\title{
Does compulsory vaccination limit personal freedom? Ethical issues
}

Mariano Paternoster ${ }^{1}$, Riccardo Scotto ${ }^{2}$, Faiz Ur Rehman Saleem ${ }^{1}$, Antonio Riccardo Buonomo ${ }^{2}$,

Nicola Schiano Moriello², Claudia Casella1, Emanuele Capasso ${ }^{1}$, Salvatore Nappa ${ }^{2}$, Ivan Gentile ${ }^{2}$, Guglielmo Borgia ${ }^{2}$, and Vincenzo Graziano ${ }^{1}$

1. Department of Advanced Biomedical Sciences, University of Naples Federico II, Italy

2. Department of Clinical Medicine and Surgery - Section of Infectious Diseases, University of Naples

Federico II, Italy

\section{REVIEW}

Please cite this paper as: Paternoster M, Scotto R, Saleem FUR, Buonomo AR, Moriello NS, Casella C, Capasso E, Nappa S, Gentile I, Borgia G, Graziano V. Does compulsory vaccination limit personal freedom? Ethical issues. AMJ 2018;11(9):459-464.

https://doi.org/10.21767/AMJ.2018.3462

\section{Corresponding Author:}

Riccardo Scotto MD

Department of Clinical Medicine and Surgery - Section of Infectious Diseases, University of Naples Federico II,

Via Sergio Pansini 5. 80131. Naples, Italy

Email: riccardo.scotto@unina.it

\section{ABSTRACT}

\section{Background}

Despite vaccinations are scientifically proven to be safe and effective public controversies limit their application in many countries.

\section{Aims}

Aim of this review is to provide an overview of biological effects of vaccination and a picture of the ethical dilemmas about compulsory vaccination.

\section{Methods}

We conducted a review on the literature about the subject. Recent news were also included.

\section{Results}

Vaccines are the best weapon against many infectious diseases. The spread of false beliefs among people have led the government authorities to increase compulsory vaccination in order to embank new outbreaks of preventable infectious diseases.

\section{Conclusion}

Even if compulsory is quite drastic approach it could be the on only way to reach an adequate coverage and protect immunoexpressed subjects.

\section{Key Words}

Vaccination, infectious diseases, epidemiology

\section{What this review adds:}

\section{What is known about this subject?}

Vaccination is worldly known to be an extremely important practice to prevent epidemic spread of infectious diseases, however in many countries vaccination coverage remains inadequate.

\section{What new information is offered in this review?}

This review explores the biological bases of vaccination and starting from an historical prospective analyses argument for and against compulsory vaccination.

3. What are the implications for research, policy, or practice?

An improvement of communication about vaccination is needed, compulsory vaccination could be the only way to reach optimal coverage.

\section{Introduction}

The term vaccine refers to biological preparations produced from living microorganisms or synthetic products that enhance immunity preventing specific infectious diseases. ${ }^{1}$ To date, they are considered as one of the greatest public health successes in the history of medicine. Indeed, the realization of mass vaccination programs has significantly reduced morbidity and mortality from most bacterial and viral infections. ${ }^{2}$ Despite their success, most vaccines have been developed empirically, with little or no understanding 
of the immunological mechanisms at the base of their effectiveness. ${ }^{3}$ Vaccines are composed of either an entire microorganism or some of its components. They may be assembled in several ways: from living microorganisms that have been weakened (live attenuated vaccines); from whole microorganisms that have been inactivated by chemical, thermal, or other methods; from molecular components of the microorganisms, such as specific proteins, polysaccharides, or nucleic acids; from inactivated toxins (toxoids) of toxin-producing bacteria; from the linkage (conjugation) of polysaccharides to proteins able to increase the effectiveness of the vaccine. ${ }^{4}$ Moreover, some vaccines include, in a single formulation, components from different disease-causing microorganisms or from several serotypes of a single microorganism, providing protection against different microorganisms or different serotypes of a single microorganism, respectively.

Live attenuated vaccines elicit strong cellular and antibody responses and often confer immunity that lasts for several decades, with even a single immunization, whereas nonliving vaccines habitually induce protection of much shorter duration and require booster vaccination to maintain protective immunity. ${ }^{1,5}$ Furthermore, the latter usually require the addition, in the vaccine formulation, of substances called adjuvants that enhance and modulate the immune response induced by the vaccine antigens. ${ }^{6}$ The most commonly used adjuvant for vaccines is aluminum salt. ${ }^{7}$

Vaccines are believed to confer protection through the induction of a memory immunity. ${ }^{8}$ This is the result of a complex immune response.

The latency between the primary antigen exposure and the activation and differentiation into effector and memory $T$ and B-cells (primary antibody response) is, commonly, of 710 days. This period, also called "lag phase", is followed by a "logarithmic phase" characterized by an increase in serum antibody levels that has classically a logarithmic pattern. The successive phase is the "plateau phase" in which after a variable period of maintenance of peak antibody levels, there is a reduction in serum antibody levels. Thanks to the development of memory $B$ and $T$ cells, the latency between a successive exposure to the antigen and development of the immune response will be shorter (usually 1 to 3 days for the lag phase). This will allow the immune system to contain and eliminate the infection before it can cause any damage and represent the main mechanism by which vaccines are effective. ${ }^{9}$ However, the effectiveness and the response duration of a vaccine depends both on the ability of the vaccine's constituents to elicit the immune response both on the characteristics of the diseases-causing microorganisms. For example, some viruses (e.g., the influenza virus), change every year, requiring annual immunization against new circulating strains; ${ }^{10}$ on the other hand, other viruses (e.g., HIV), escape the immune system making very difficult the formulation of an effective vaccine. $^{11}$

Even though any infectious disease might be preventable with a vaccine, the limited understanding of all the immune mechanisms involved and the high variability of the immune response to each specific microorganism, have so far limited the development of vaccines to a close number of viral and bacterial diseases. Nonetheless, vaccines represent the most cost-effective life-saving medication in history. At present, human vaccines are used in the prevention of more than thirty infectious diseases and are worldwide employed in public health programs. Consequently, infections that were cause of loss of health and life a few decades ago are now rarely seen. More than 100 million children are vaccinated annually against diseases such as diphtheria, tetanus, pertussis, tuberculosis, polio, measles, and hepatitis $B$. It is estimated that vaccinations prevent about 2.5 million deaths each year. ${ }^{12,13}$

\section{The Immunity Cycle}

In natural settings, populations usually undergo cyclical changes in immunity; such phenomenon is known as "The Immunity cycle". The cycle begins with the introduction of a disease, usually from the zoonotic pool. ${ }^{14,15}$ Entering a new population, many individuals within the population quickly succumb to the new disease, as a result of a lack of immunity, overall increasing the mortality levels in a "mortality phase". However small groups or more resilient demographics among the populations are able to develop some form of immunity and can survive without infection or, alternatively, they survive the infection and develop immunity. The population as a whole begins to either develop a level of immunity through adaptive immunity or herd immunity resulting in a "revival phase" with a decline in mortality rates. ${ }^{16}$ It should be noted that in some circumstances the revival phase might be due to a decrease in microbial traffic.

The surviving Individuals gain some form immunity, survive and reproduce. After some amount of time, the population begins to benefit from a combination of active, passive and herd immunity in a phase, which can be described as the "Intermediate Immunity period" here immunological memory prevents the rise of an epidemic. Where 
immediate generations either acquire active immunity themselves or benefit from the immunity of others through herd immunity, while successive generations benefit through passive and herd immunity. The intermediate immunity phase can be identified by mortality rate, which should return to low levels in this period.

However, as generations pass the immunity of the population falters with many individuals loosing passive immunity and compromising the existing herd immunity.

Eventually, the existing equilibrium reaches a stage where the population is no longer immune and the number of individuals who maintain a level of immunity are too few to provide any sort of herd immunity. At this stage, known as "The susceptibility Period", the population becomes almost destined to another epidemic. The overall trend can ideally be seen in the 1630 plague. $^{17}$

These trends are particularly prominent in the cyclical epidemics often seen in a variety of diseases especially throughout the eighteenth and ninetieth centuries. ${ }^{18}$

From the cycle, it can be understood that vaccines form a pivotal role by prolonging the immunity period indefinitely and thereby reducing deaths. It is crucial that all individuals in society are vaccinated to permanently stop the cycle. Vaccines are essential in this context; parents who refuse to vaccinate their children by many regards allow the cycle to continue and affect many others in the future.

An example of this was seen when during the 1970s the adverse effect of the pertussis vaccine was disproportionately publicized, the resulting decline in immunization rates (decreasing from 90-80 per cent to 30 per cent) resulted in two serious outbreaks of whooping cough. ${ }^{19}$ It is therefore necessary to vaccinate all children and adults who do not present contraindication.

\section{Compulsory vaccination to increase vaccination coverage}

Vaccination is compulsory in many countries since 1800 . To obtain an adequate vaccination coverage, vaccination policies were introduced in Great Britain in the first half of the XIX century with the vaccination acts of 1840, 1841 and 1853 that maid smallpox vaccination free and sequentially compulsory.

In Italy, smallpox vaccination was first suspended in 1977 and then abolished in 1981. In the meantime, however, several other vaccinations like diphtheria (1939), polio
(1966), tetanus (1968) and B hepatitis (1991) were made mandatory.

Other countries like France, Greece, Portugal and Belgium opted for compulsory vaccination too, while, in countries like the United Kingdom and Finland, there are no mandatory vaccines. However, in these countries, there are strong incentives to promote vaccination and widespread propaganda coverage.

In Germany it was chosen a sort of middle way policy: there were no sanctions for missing vaccination, but a vaccination certificate was and is required for school admission.

\section{Public controversies on compulsory vaccinations}

"no-vax" campaigns are acquiring more space on mass media and even some political figures took a position against vaccination obligation, emphasizing the notoriously fallacious association between vaccination and autism. These positions are confusing public opinion giving straight to "no-vax" thesis.

By consequence, people do not understand effectiveness and safety of vaccinations that are perceived as outdated arguments or, even worse, as an artifice in favor of pharmaceutical companies. $^{20}$

A putative correlation between trivalent vaccination and autism, was first theorized in Andrew Wakefield's publications, these results were however based on maliciously falsified data, and Wakefield himself was sentenced and banned from the medical profession. ${ }^{21}$

Since Wakefield's study, the association between vaccination and autism has been denied by more than 40 publications collected by American Academy of Pediatrics. ${ }^{22-}$ ${ }^{24}$ This false correlation is not the only claim made by "novax", who are supporting freedom of choice as an absolute value and are strongly critical between the interaction of vaccines and the immune system. They maintain that vaccination may in some way overloads the immune system (nowadays it is clear that this claim has no scientific relevance). ${ }^{25}$

By contrast, several studies proof that vaccination is the best weapon against many infectious diseases. There are, indeed, strong data in support of this statement. For example, after the introduction of specific vaccination, mortality for diseases like polio, diphtheria, smallpox, rubella, congenital rubella, measles, and mumps dropped of nearly 100 per cent with positive effects on morbidity and 
quality of life. $^{19}$

Despite these overwhelming evidences, in Italy as in Europe and the United States, has grown a stance of mistrust and reluctance leading to a substantial decrease in vaccination coverage.

\section{Effects of public controversies on vaccination coverage and on health policy}

Measles is an iconic case of how these public controversies affected vaccination coverage. ${ }^{26}$

European Center for Disease prevention and Control has, indeed, recently published an epidemiologic update on measles spread in Europe between 1 July 2016 and 30 June $2017,{ }^{27}$ showing 10,886 new cases of measles, the majority of affected people had not been previously vaccinated (8742 cases, 86 per cent), while 9 per cent received just one dose of vaccine and 3 per cent received two or more doses. The proportion of not vaccinated subjects is higher among children of less than 1 year, who cannot yet be vaccinated and rely entirely on herd immunity, reaching 95 per cent of total cases.

These data show a substantial increase in new infections compared to the period from October 2014 to September 2015 when 4,202 new cases occurred. Once again children with less than one year of age where more affected.

In Italy, data from the ministry of health state that the vaccination coverage is 87.26 per cent ${ }^{28}$ and that there have been 4689 new cases during the first three months of 2017 whit an increase of 230 per cent compared to the same period of the previous year. ${ }^{29}$ To curb such phenomenon, European states took a series of measures to increase vaccine coverage, adopting more or less authoritarian strategies to obtain this goal. One possible strategy is the imposition of some specific vaccinations. In particular in Italy, At first, the "National Plan for Vaccine Prevention" implemented free offer of not only compulsory vaccination, but also suggested vaccination. ${ }^{30}$

Also, the "Federation of Medical Doctors and dental practitioners" acted presenting a document with 15 proposals stating that among other things; the suggestion of not vaccinating is a deontological infringement with possible disbarment as sanction. Attention has also been drawn on methods of communication, with the suggestion to avoid pre-printed form and prefer oral communication. ${ }^{31}$

The Italian Government recently passed a decree containing many actions to increase vaccine coverage. This decree, which takes into account suggestions from European and International sources, obliges to the following vaccinations: anti-diphtheria, anti-polio, anti-tetanus, anti-hepatitis B, anti-pertussis, anti-Hemophilus Influenzae type B, Antimeasles, anti-rubella, anti-mumps, anti-chickenpox. Those vaccinations can be omitted or delayed only in case of documented health hazard certified by a medical doctor.

\section{Discussion}

The decrease of vaccination coverage is a worrying phenomenon, endangering entire society. This decrease may, in fact, expose the society to almost disappeared infectious diseases. In particular, parents who refuse to vaccinate their children are afraid of the potential dangers of vaccination, ignoring instead the real danger represented by infectious diseases that are mistakenly thought as disappeared. $^{32}$ By contrast, medical doctors should counteract this erroneous belief. To not vaccinate should be indeed seen as selfish gesture jeopardizing herd immunity and exposing immunosuppressed subjects and future generation to the risk of epidemics. ${ }^{33}$

Vaccines are especially targeted to children; this protection falls into parent's duty and cannot be regarded as a form of "freedom of choice on the basis of the priority of the interests of children and his right to be vaccinated".

Public opinion regarding state promoted immunization programs often recalls the previously cited immunity cycle; this phenomenon can be considered as cycle of public perception based on immunity. That is to say that when mortality from an infectious disease rises people ask for the availability of a vaccine that is given to lots of subjects. However, when entering in the intermediate phase, collective memory of the disease fades and, at the same time, news about side effects of vaccination rises. This leads to a decrease in the numbers or vaccinations with possible new bursts of epidemics. Consequently, it is a priority that every news and debate on mass media should be strictly based on scientific evidenc. $^{34}$

In conclusion, vaccines are a formidable weapon against Infectious Diseases. However, their success in preventing cases and death from Infectious Diseases implies a reduced perception on the population of the danger and risk due to Infectious Diseases. This reduced perception of risk for Infectious Diseases is one of the main drivers of "no-vax" movements. Therefore, paradoxically, the reasons for antivaccination organizations lie in the very high efficacy of the vaccine in preventing infections and death. To overcome 
this "issue", we think that a potential conflict between personal freedom and public health does exist, but compulsory vaccination currently represents the only strategy to reach heard immunity levels that allow to prevent infections at a population level, even in severely immunosuppressed subjects, who are the most vulnerable part of the society, and can achieve health and prevention from infectious Diseases only in this way.

\section{References}

1. Plotkin S, Orenstein W, Offit P. Vaccines. 5th ed. Saunders/Elsevier; 2008.

2. Frieden TR, Jaffe HW, Stephens JW, et al. Centers for disease control and prevention MMWR editorial and production staff. MMWR. 2011;60.

3. Rappuoli R. From Pasteur to genomics: progress and challenges in infectious diseases. Nat Med. 2004;10:1177-85. doi:10.1038/nm1129.

4. Hansson M, Nygren PA, Ståhl S. Design and production of recombinant subunit vaccines. Biotechnol Appl Biochem. 2000;32 ( Pt 2):95-107.

5. Plotkin SA. Vaccines: past, present and future. Nat Med. 2005;10:S5-11. doi:10.1038/nm1209.

6. Lee $S$, Nguyen MT. Recent advances of vaccine adjuvants for infectious diseases. Immune Netw. 2015;15:51. doi:10.4110/in.2015.15.2.51.

7. Coffman RL, Sher A, Seder RA. Vaccine adjuvants: Putting innate immunity to work. Immunity. 2010;33:492-503. doi:10.1016/j.immuni.2010.10.002.

8. Plotkin SA. Vaccines: Correlates of vaccine-induced immunity. Clin Infect Dis. 2008;47:401-9. doi:10.1086/589862.

9. Institute of Medicine (U.S.). Committee to review adverse effects of vaccines., Stratton K. Adverse effects of vaccines : evidence and causality. National Academies Press; 2012.

10. Han T, Marasco WA. Structural basis of influenza virus neutralization. Ann N Y Acad Sci. 2011;1217:178-90. doi:10.1111/j.1749-6632.2010.05829.x.

11. Pulendran B, Ahmed R. Immunological mechanisms of vaccination. Nat Immunol. 2011;12:509-17.

12. WHO | State of the world's vaccines and immunization. Third edition. WHO 2013.

13. Barnighausen T, Bloom DE, Cafiero-Fonseca ET, et al. Valuing vaccination. Proc Natl Acad Sci. 2014;111:12313-9. doi:10.1073/pnas.1400475111.

14. Morse SS. Factors in the emergence of infectious diseases. Emerg Infect Dis. 1995;1:7-15. doi:10.3201/eid0101.950102.

15. Morse SS. Emerging viruses: defining the rules for viral traffic. Perspect Biol Med. 1991;34:387-409.
16. Rahaman MM, Khan MM, Aziz KM, et al. An outbreak of dysentery caused by Shigella dysenteriae type 1 on a coral island in the Bay of Bengal. J Infect Dis. 1975;132:15-9.

17. $\mathrm{CMH}$ annual reports 1998. http://www.history.ac.uk/cmh/arpt89.html (accessed March 31, 2018).

18. Rohmer L, Jacobs MA, Brittnacher MJ, et al. Genomic analysis of the emergence of 20th century epidemic dysentery. BMC Genomics. 2014;15:355. doi:10.1186/1471-2164-15-355.

19. Ulmer JB, Liu MA. Ethical issues for vaccines and immunization. Nat Rev Immunol. 2002;2:291-6. doi:10.1038/nri780.

20. Chervenak FA, McCullough LB, Brent RL. Professional responsibility and early childhood vaccination. J Pediatr. 2016;169:305-9. doi:10.1016/j.jpeds.2015.10.076.

21. Pellizzone A. II caso Wakefield: ecco com'è andata a finire. Epic ISS n.d. http://www.epicentro.iss.it/leggere/wakefield.asp (accessed March 31, 2018).

22. Taylor LE, Swerdfeger AL, Eslick GD. Vaccines are not associated with autism: An evidence-based metaanalysis of case-control and cohort studies. Vaccine. 2014;32:3623-9. doi:10.1016/j.vaccine.2014.04.085.

23. Gentile I, Bravaccio C, Bonavolta R, et al. Response to measles-mumps-rubella vaccine in children with autism spectrum disorders. In Vivo. 2013;27:377-82.

24. American Academy of Pediatrics. Vaccine Safety: Examine the Evidence 2013.

25. 6 dangerous anti-vaccination arguments analyzed, explained, and shut down. Huffingt POST n.d. https://www.huffingtonpost.com/2015/02/05/antivaccine-arguments-analyzed-explained_n_6607026.html (accessed April 1, 2018).

26. Jamrozik E, Handfield T, Selgelid MJ. Victims, vectors and villains: are those who opt out of vaccination morally responsible for the deaths of others? J Med Ethics. 2016;42:762-8. doi:10.1136/medethics-2015-103327.

27. Communicable disease threats report, 4 June - 10 June 2017, week 23. ECDC Commun Dis Threat Rep. 2017. https://ecdc.europa.eu/en/publicationsdata/communicable-disease-threats-report-4-june-10june-2017-week-23 (accessed April 1, 2018).

28. Copertura vaccinale in Italia. Epic ISS n.d. http://www.epicentro.iss.it/temi/vaccinazioni/dati_Ita.a sp (accessed April 1, 2018).

29. Measles Weekly Report. 2017.

30. Piano Nazionale Prevenzione Vaccinale 2017-2019. 2017.

31. Attena F, Valdes Abuadili A, Marino S. The informed 
consent in Southern Italy does not adequately inform parents about infant vaccination. BMC Public Health. 2014;14:211. doi:10.1186/1471-2458-14-211.

32. Weithorn LA, Reiss DR. Legal approaches to promoting parental compliance with childhood immunization recommendations. Hum Vaccin Immunother. 2018:1-8. doi:10.1080/21645515.2018.1423929.

33. Sim FM. Individualism and social solidarity in vaccination policy: some further considerations. Isr J Health Policy Res. 2017;6:21. doi:10.1186/s13584-017-0147-2.

34. Baker JP. Immunization and the American way: 4 childhood vaccines. Am J Public Health. 2000;90:199207.

\section{PEER REVIEW}

Not commissioned. Externally peer reviewed.

\section{CONFLICTS OF INTEREST}

The authors declare that they have no competing interests.

\section{FUNDING}

None

\section{ETHICS COMMITTEE APPROVAL}

Not applicable 